Appl. Set-Valued Anal. Optim. 3 (2021), No. 3, pp. 317-323

Available online at http://asvao.biemdas.com

https://doi.org/10.23952/asvao.3.2021.3.06

\title{
CONTINUITY OF MAXIMAL VALUE FUNCTIONALS IN NORMED SPACES
}

\author{
JOHANNES JAHN \\ Department Mathematik, Universität Erlangen-Nürnberg, Cauerstr. 11, 91058 Erlangen, Germany
}

\begin{abstract}
This paper examines special maximal value functionals, which are extended support functionals where the objective is linear but the constraint set is nonconvex. In finite-dimensional spaces, it is well-known that these maximal value functionals are continuous. It is shown in the present paper that this result can also be proven in normed spaces without convexity assumptions. As an application, necessary optimality conditions in set optimization are derived.
\end{abstract}

Keywords. Necessary optimality condition; Maximal value functional; Set optimization.

\section{INTRODUCTION}

Support functionals play an important role in convex analysis and parametric optimization. Under appropriate assumptions, it is a known result in finite dimensional spaces that support functionals are continuous (e.g., compare [1, Prop. 4.4] and [2, Lemma 2.1]).

In general, the proof of such a result is based on the finite dimensional structure of the space. Extensions of this result to infinite dimensional Banach spaces are known. For instance, it was stated by Borwein and Vanderwerff [3, Prop. 4.1.5] that the support functional of a nonempty closed convex set is continuous on the interior of its domain (compare [4], [5], and [6]). In the present paper, we investigate an extension of a support functional, simply called maximal value functional.

For a maximal value functional, the objective is linear. We do not assume the convexity of the constraint set, and its domain is a nonempty weakly compact subset of the topological dual space of a normed space. We present a direct proof of the continuity of the maximal value functional without the use of convex analysis. Such a specific approach has an important application in set optimization.

Although several authors investigated the assumptions under which a support functional is even Gâteaux or Fréchet differentiable (compare [4], [5], and [6]), we are only interested in the issue of continuity. This is helpful for the classification of Lagrange multipliers in set optimization. Since maximal value functionals belong to the real linear space of real-valued continuous functions, and its dual space is well-known, Lagrange multipliers in set optimization problems are finite signed Radon measures with special properties.

E-mail address: johannes.jahn@fau.de.

Received November 26, 2020; Accepted March 30, 2021.

(C)2021 Applied Set-Valued Analysis and Optimization 
First steps to an extension of the continuity result for maximal value functionals to the infinite dimensional case were done in [7]. With the new result of the present paper, the KarushKuhn-Tucker theory in set optimization developed in [2] can be simply extended to reflexive Banach spaces (also compare [7]). Since set optimization problems can be transformed to vector optimization problems (see [8]) where maximal and minimal value functionals are the decisive key, set optimization is an important application of maximal value functionals.

Following the concept of weakly minimal elements of a set in vector optimization, the new optimality notion of a weak minimal solution is introduced in set optimization. This notion is based on maximal value functionals. The presented optimality condition is necessary for weak minimal solutions.

This paper is organized as follows. After this short introduction the main result on the continuity of maximal value functionals is presented in Section 2. The problems of set optimization together with a necessary optimality condition of weak minimal solutions are given in Section 3 , the last section.

\section{MAin Results}

For the main result on the continuity of a maximal value functional, we use the following assumption.

Assumption 2.1. Let $A$ be a nonempty weakly compact subset of a real normed space $\left(Y,\|\cdot\|_{Y}\right)$, and let $T^{*}$ be a nonempty weakly compact subset of the topological dual space $\left(Y^{*},\|\cdot\|_{Y^{*}}\right)$.

Under this assumption, we investigate the maximal value functional $\varphi: T^{*} \rightarrow \mathbb{R}$ with

$$
\ell \mapsto \varphi(\ell)=\max _{y \in A} \ell(y) .
$$

By the Weierstraß theorem, for all $\ell \in T^{*}$, this appearing optimization problem has a solution. Therefore, the functional $\varphi$ is well-defined.

The set $A$ is not assumed to be convex but if it is convex, then the functional $\varphi$ is also called a support functional.

Theorem 2.1. Under Assumption 2.1, the maximal value functional $\varphi$ is continuous on $T^{*}$.

Proof. Let $\left(\ell_{n}\right)_{n \in \mathbb{N}}$ be an arbitrary norm convergent sequence in $T^{*}$ with limit $\bar{\ell} \in T^{*}$. We now prove the assertion by various parts.

(a) Because the set $A$ is weakly compact, we have that $A$ is norm bounded by some $\alpha \geq 0$ (compare [9, p. 17]). Since norm convergent sequences are bounded, there exists some $\beta \geq 0$ with

$$
\left\|\ell_{n}\right\|_{Y^{*}} \leq \beta \text { for all } n \in \mathbb{N}
$$

Consequently, for every $n \in \mathbb{N}$, there exists some $y_{n} \in A$ such that

$$
\left|\varphi\left(\ell_{n}\right)\right|=\left|\ell_{n}\left(y_{n}\right)\right| \leq\left\|\ell_{n}\right\|_{Y^{*}}\left\|y_{n}\right\|_{Y} \leq \beta \alpha .
$$

By the Bolzano-Weierstraß theorem, $\liminf _{n \rightarrow \infty} \varphi\left(\ell_{n}\right)$ and $\lim _{\sup _{n \rightarrow \infty}} \varphi\left(\ell_{n}\right)$ then exist.

(b) Since $\limsup _{n \rightarrow \infty} \varphi\left(\ell_{n}\right)$ exists, there is a subsequence $\left(\ell_{n_{k}}\right)_{k \in \mathbb{N}}$ with $\lim _{k \rightarrow \infty} \varphi\left(\ell_{n_{k}}\right)=\limsup _{n \rightarrow \infty} \varphi\left(\ell_{n}\right)$.

Because the set $A$ is weakly compact, there is a subsequence $\left(y_{n_{k_{r}}}\right)_{r \in \mathbb{N}}$ in $A$ converging weakly 
to some $\bar{y} \in A$. Because of the norm convergence of $\left(\ell_{n_{k r}}\right)_{r \in \mathbb{N}}$ to $\bar{\ell}$ and the weak convergence of $\left(y_{n_{k}}\right)_{r \in \mathbb{N}}$ to $\bar{y}$, we obtain $\lim _{r \rightarrow \infty} \ell_{n_{k_{r}}}\left(y_{n_{k_{r}}}\right)=\bar{\ell}(\bar{y})$ (compare [10, Thm. 7.1.5,(vi)]). Hence,

$$
\limsup _{n \rightarrow \infty} \varphi\left(\ell_{n}\right)=\lim _{r \rightarrow \infty} \varphi\left(\ell_{n_{k_{r}}}\right)=\lim _{r \rightarrow \infty} \ell_{n_{k_{r}}}\left(y_{n_{k_{r}}}\right)=\bar{\ell}(\bar{y}) \leq \varphi(\bar{\ell}) .
$$

(c) Because of the weak compactness of the set $A$, there exists some $\hat{y} \in A$ with $\bar{\ell}(\hat{y})=\varphi(\bar{\ell})$. Since $\liminf _{n \rightarrow \infty} \varphi\left(\ell_{n}\right)$ exists by part (a), there exits a subsequence $\left(\ell_{n_{m}}\right)_{m \in \mathbb{N}}$ with $\lim _{m \rightarrow \infty} \varphi\left(\ell_{n_{m}}\right)=$ $\liminf _{n \rightarrow \infty} \varphi\left(\ell_{n}\right)$. Because the sequence $\left(\ell_{n_{m}}\right)_{m \in \mathbb{N}}$ is norm convergent, it is also weak* convergent and we obtain $\lim _{m \rightarrow \infty} \ell_{n_{m}}(\hat{y})=\bar{\ell}(\hat{y})$. Consequently,

$$
\liminf _{n \rightarrow \infty} \varphi\left(\ell_{n}\right)=\lim _{m \rightarrow \infty} \varphi\left(\ell_{n_{m}}\right) \geq \lim _{m \rightarrow \infty} \ell_{n_{m}}(\hat{y})=\bar{\ell}(\hat{y})=\varphi(\bar{\ell}) .
$$

(d) Inequalities (2.1) and (2.2) imply

$$
\limsup _{n \rightarrow \infty} \varphi\left(\ell_{n}\right) \leq \varphi(\bar{\ell}) \leq \liminf _{n \rightarrow \infty} \varphi\left(\ell_{n}\right) .
$$

Hence,

$$
\lim _{n \rightarrow \infty} \varphi\left(\ell_{n}\right)=\varphi(\bar{\ell})
$$

This completes the proof.

Theorem 2.1 means that the maximal value functional $\varphi$ belongs to the real linear space $\mathscr{C}\left(T^{*}\right)$ of real-valued continuous functions defined on the weakly compact set $T^{*}$. This space has a known dual space $\mathscr{C}^{*}\left(T^{*}\right)$, which consists of all finite signed Radon measures. The knowledge of this dual space is helpful in applications.

It is stated by Clarke [11, Prop. 2.2.6] that under a convexity assumption the maximal value functional $\varphi$ is locally Lipschitz continuous if $\varphi$ is bounded above on a neighborhood of some element of an open subset $\tilde{T}^{*}$ of the set $T^{*}$. Recall that the maximal value functional $\varphi$ is said to be Lipschitz continuous near some $\bar{\ell} \in \tilde{T}^{*}$ iff there is an open ball $B(\bar{\ell}, \varepsilon):=\left\{\ell \in Y^{*} \mid \| \ell-\right.$ $\left.\bar{\ell} \|_{Y^{*}}<\varepsilon\right\}$ for some $\varepsilon>0$ and a Lipschitz constant $K>0$ with the property

$$
\left|\varphi\left(\ell_{1}\right)-\varphi\left(\ell_{2}\right)\right| \leq K\left\|\ell_{1}-\ell_{2}\right\|_{Y^{*}} \text { for all } \ell_{1}, \ell_{2} \in \tilde{T}^{*} \cap B(\bar{\ell}, \varepsilon) .
$$

Proposition 2.1 ([11, Prop. 2.2.6]). Let Assumption 2.1 be satisfied. In addition, let the set $\tilde{T}^{*}$ be a nonempty convex open subset of $T^{*}$, and let the maximal value functional $\varphi$ be bounded above on a neighborhood of some element of $\tilde{T}^{*}$. Then $\varphi$ is Lipschitz continuous near any $\ell \in \tilde{T}^{*}$.

This proposition follows from [11, Prop. 2.2.6] because the maximal value functional is convex on the convex set $\tilde{T}^{*}$.

Recall that the functional $\varphi: T^{*} \rightarrow \mathbb{R}$ has a subgradient at some $\bar{\ell} \in T^{*}$ iff there exists some continuous linear functional $L: Y^{*} \rightarrow \mathbb{R}$ with

$$
\varphi(\ell) \geq \varphi(\bar{\ell})+L(\ell-\bar{\ell}) \text { for all } \ell \in T^{*} .
$$

Corollary 2.1. Let Assumption 2.1 be satisfied. In addition, let the set $T^{*}$ be convex and let it have a nonempty interior. Then the functional $\varphi$ has a subgradient at every $\bar{\ell} \in \operatorname{int}\left(T^{*}\right)$.

Proof. By Theorem 2.1, the functional $\varphi$ is continuous on $T^{*}$. It is obvious that the maximal value functional $\varphi$ is convex. By a known result of convex analysis (compare [12, Thm. 3.26]), the functional $\varphi$ has a subgradient at every $\bar{\ell} \in \operatorname{int}\left(T^{*}\right)$. 


\section{The Application to Set Optimization}

The optimality conditions for problems of set optimization were investigated in [2] in a finite dimensional setting. It is obvious from [7] that the Karush-Kuhn-Tucker theory in [2] can be extended to the infinite-dimensional case. Now, we present the results for the notion of a weak minimal solution.

In this section, set optimization problems are considered under the following non-standard assumption.

Assumption 3.1. Let $S$ be a nonempty subset of a real linear space $X$, and let $\left(Y,\|\cdot\|_{Y}\right)$ be a real reflexive Banach space partially ordered by a closed convex cone $C$. Let $F: S \rightrightarrows Y$ be a set-valued map. For every $x \in S$, let the set $F(x)$ be nonempty, convex and weakly compact.

Then, we investigate the set optimization problem

$$
\min _{x \in S} F(x),
$$

where $S$ denotes the constraint set, and the objective map $F$ is set-valued. There are various order relations (see [13]), which can be used for the definition of a solution of problem (3.1). We start with the well-known set less order relation introduced by Young [14] in 1931.

Definition 3.1. For nonempty subsets $A, B$ of a real linear space $Y$ partially ordered by a convex cone $C$ the set less order relation $\preccurlyeq$ is defined by

$$
A \preccurlyeq B: \Longleftrightarrow B \subset A+C \text { and } A \subset B-C .
$$

Under Assumption 3.1, this order relation can be characterized by the following proposition if we introduce the set

$$
T^{*}:=C^{*} \cap\left\{\ell \in Y^{*} \mid\|\ell\|_{Y^{*}} \leq 1\right\}
$$

where $C^{*}:=\left\{\ell \in Y^{*} \mid \ell(c) \geq 0\right.$ for all $\left.c \in C\right\}$ denotes the dual cone of $C$. Since the closed unit ball in the dual space of a reflexive Banach space is weakly compact (compare [10, Lemma 7.2.2 (iv) and Thm. 7.2.4]), and the dual cone $C^{*}$ is convex and closed and thus also weakly closed, the set $T^{*}$ is weakly compact.

Proposition 3.1 ([7, Satz 3.10], [8, Thm. 2.1]). Let Assumption 3.1 be satisfied, and let $x_{1}, x_{2} \in S$ be arbitrarily chosen. Then

$$
\begin{aligned}
& F\left(x_{1}\right) \preccurlyeq F\left(x_{2}\right) \Longleftrightarrow \forall \ell \in T^{*} \backslash\left\{0_{Y^{*}}\right\}: \min _{y \in F\left(x_{1}\right)} \ell(y) \leq \min _{y \in F\left(x_{2}\right)} \ell(y) \text { and } \\
& \max _{y \in F\left(x_{1}\right)} \ell(y) \leq \max _{y \in F\left(x_{2}\right)} \ell(y) .
\end{aligned}
$$

Proof. Let $x_{1}, x_{2} \in S$ be arbitrarily chosen. The sets $F\left(x_{1}\right)+C$ and $F\left(x_{2}\right)+C$ are convex and weakly closed and thus also closed. Moreover, the optimization problems $\min _{y \in F\left(x_{1}\right)} \ell(y)$, $\min _{y \in F\left(x_{2}\right)} \ell(y), \max _{y \in F\left(x_{1}\right)} \ell(y)$ and $\max _{y \in F\left(x_{2}\right)} \ell(y)$ are solvable for all $\ell \in T^{*}$. Then [7, Satz 3.10] (see also [8, Thm. 2.1]) is applicable, and the assertion follows immediately.

The equivalence in (3.2) motivates the following definition of a strong set less order relation, which is then used for the definition of a weak minimal solution.

Definition 3.2. Let Assumption 3.1 be satisfied. 
(a) For arbitrary $x_{1}, x_{2} \in S$, the strong set less order relation $\prec$ is defined by

$$
\begin{array}{r}
F\left(x_{1}\right) \prec F\left(x_{2}\right) \Longleftrightarrow \forall \ell \in T^{*} \backslash\left\{0_{Y^{*}}\right\}: \min _{y \in F\left(x_{1}\right)} \ell(y)<\min _{y \in F\left(x_{2}\right)} \ell(y) \text { and } \\
\max _{y \in F\left(x_{1}\right)} \ell(y)<\max _{y \in F\left(x_{2}\right)} \ell(y) .
\end{array}
$$

(b) An element $\bar{x} \in S$ is called a weak minimal solution of the optimization problem (3.1) iff

$$
\nexists x \in S \text { with } F(x) \neq F(\bar{x}): F(x) \prec F(\bar{x}) .
$$

The notion of a weak minimal solution is an extension of the notion of weakly minimal elements in vector optimization (compare [15, Def. 4.12]). In Definition 3.2, (b) the set $F(\bar{x})$ could also be interpreted as a weakly minimal element of the set system $\{F(x) \mid x \in S\}$.

Before, we present a necessary optimality condition for a weak minimal solution of set optimization problem (3.1), we recall the notion of a directional variation, which generalizes the well-known notion of a directional derivative.

Definition 3.3 ([15, Def. 2.14]). Let $A$ be a nonempty subset of a real linear space $U$, and let $B$ be a nonempty subset of a real linear space $V$. Moreover, let a map $g: A \rightarrow V$ and an element $\bar{a} \in A$ be given. A map $g^{\prime}(\bar{a}): A-\{\bar{a}\} \rightarrow V$ is called a directional variation of $g$ at $\bar{a}$ with respect to $B$ iff the following holds: Whenever there is an element $a \in A$ with $a \neq \bar{a}$ and $g^{\prime}(\bar{a})(a-\bar{a}) \in B$, then there is a $\bar{\lambda}>0$ with

$$
\bar{a}+\lambda(a-\bar{a}) \in A \text { for all } \lambda \in(0, \bar{\lambda}]
$$

and

$$
\frac{1}{\lambda}(g(\bar{a}+\lambda(a-\bar{a}))-g(\bar{a})) \in V \text { for all } \lambda \in(0, \bar{\lambda}]
$$

Moreover, we need the notion of a convex-like map. With [15, Thm. 2.11], a map, which maps a nonempty subset of a real linear space to a partially ordered real linear space, is convexlike iff the algebraic sum of its image set and the convex cone defining the partial order in the image space is a convex set. This notion extends the notion of a convex map.

Theorem 3.1. Let Assumption 3.1 be satisfied, and let $\bar{x} \in S$ be a weak minimal solution of the set optimization problem (3.1). Moreover, let the functions $\varphi_{-}, \varphi_{+}: S \rightarrow \mathscr{C}\left(T^{*}\right)$ defined by

$$
\varphi_{-}(x)(\ell):=\max _{y \in-F(x)} \ell(y) \text { for all } \ell \in T^{*}
$$

and

$$
\varphi_{+}(x)(\ell):=\max _{y \in F(x)} \ell(y) \text { for all } \ell \in T^{*}
$$

have a directional variation $\varphi_{-}^{\prime}$ and $\varphi_{+}^{\prime}$, respectively, at $\bar{x}$ with respect to $-\operatorname{int}\left(\mathscr{C}_{+}\left(T^{*}\right)\right)$, where

$$
\mathscr{C}_{+}\left(T^{*}\right):=\left\{\psi \in \mathscr{C}\left(T^{*}\right) \mid \psi(\ell) \geq 0 \forall \ell \in T^{*}\right\}
$$

denotes the natural order cone in $\mathscr{C}\left(T^{*}\right)$. Then, for every $x \in S$, there exits some $\bar{\ell} \in T^{*}$ with

$$
\max \left\{-\varphi_{-}^{\prime}(\bar{x})(\bar{\ell})(x-\bar{x}), \varphi_{+}^{\prime}(\bar{x})(\bar{\ell})(x-\bar{x})\right\} \geq 0 .
$$


If, in addition, the directional variation of the map $f: S \rightarrow \mathscr{C}^{2}\left(T^{*}\right)$ at $\bar{x}$ with

$$
f(x)(\ell)=\left(\begin{array}{r}
-\varphi_{-}(x)(\ell) \\
\varphi_{+}(x)(\ell)
\end{array}\right) \text { for all } x \in S \text { and } \ell \in T^{*}
$$

is convex-like, then there exist finite signed Radon measures $\mu_{1}, \mu_{2} \in \mathscr{C}_{+}^{*}\left(T^{*}\right)$ with $\left(\mu_{1}, \mu_{2}\right) \neq$ $\left(0_{\mathscr{C}^{*}\left(T^{*}\right)}, 0_{\mathscr{C}^{*}\left(T^{*}\right)}\right)$ and

$$
-\int_{T^{*}} \varphi_{-}^{\prime}(\bar{x})(\cdot)(x-\bar{x}) d \mu_{1}+\int_{T^{*}} \varphi_{+}^{\prime}(\bar{x})(\cdot)(x-\bar{x}) d \mu_{2} \geq 0 \text { for all } x \in S .
$$

Proof. Let $\bar{x} \in S$ be a weak minimal solution of set optimization problem (3.1). The proof of the necessary optimality conditions is divided into several parts.

(a) By Definition 3.2, it is obvious that $\bar{x}$ is a weak minimal solution of the vector optimization problem $\min _{x \in S} \tilde{f}(x)$ with $\tilde{f}: S \rightarrow \mathscr{R}^{2}\left(T^{*}\right)$ (space of functions defined on $T^{*}$ and mapping to $\overline{\mathbb{R}}^{2}$ ) given by

$$
\tilde{f}(x)(\ell)=\left(\begin{array}{c}
\min _{y \in F(x)} \ell(y) \\
\max _{y \in F(x)} \ell(y)
\end{array}\right) \text { for all } x \in S \text { and } \ell \in T^{*} .
$$

Here in the image space of $\tilde{f}$ the componentwise and pointwise order relation is used. For arbitrary $x \in S$ and $\ell \in T^{*}$, we have from (3.3) that

$$
\min _{y \in F(x)} \ell(y)=-\max _{y \in F(x)}-\ell(y)=-\max _{y \in-F(x)} \ell(y)=-\varphi_{-}(x)(\ell) .
$$

With (3.4), the vector function $\tilde{f}$ can be written as

$$
\tilde{f}(x)(\ell)=\left(\begin{array}{r}
-\varphi_{-}(x)(\ell) \\
\varphi_{+}(x)(\ell)
\end{array}\right)=f(x)(\ell) \text { for all } x \in S \text { and } \ell \in T^{*} .
$$

From Theorem 2.1, for every $x \in S$, the function $f(x)(\cdot)$ is continuous. Hence, $f: S \rightarrow \mathscr{C}^{2}\left(T^{*}\right)$. The real linear space $\mathscr{C}\left(T^{*}\right)$ is partially ordered by the convex cone $\mathscr{C}_{+}\left(T^{*}\right)$. This cone has a nonempty interior int $\left(\mathscr{C}_{+}\left(T^{*}\right)\right)$, and the dual cone of $\mathscr{C}_{+}\left(T^{*}\right)$ can be written as

$$
\mathscr{C}_{+}^{*}\left(T^{*}\right):=\left\{\mu \in \mathscr{C}^{*}\left(T^{*}\right) \mid \int_{T^{*}} \psi d \mu \geq 0 \text { for all } \psi \in \mathscr{C}_{+}\left(T^{*}\right)\right\} .
$$

(b) Since $\varphi_{-}$and $\varphi_{+}$have a directional variation at $\bar{x}$ with respect to $-\operatorname{int}\left(\mathscr{C}_{+}\left(T^{*}\right)\right)$, it follows from [15, Thm. 7.6] that

$$
\left(\begin{array}{r}
-\varphi_{-}^{\prime}(\bar{x})(\cdot) \\
\varphi_{+}^{\prime}(\bar{x})(\cdot)
\end{array}\right)(x-\bar{x}) \notin\left[-\operatorname{int}\left(\mathscr{C}_{+}\left(T^{*}\right)\right)\right] \times\left[-\operatorname{int}\left(\mathscr{C}_{+}\left(T^{*}\right)\right)\right] \text { for all } x \in S .
$$

This means, for every $x \in S$,

$$
-\varphi_{-}^{\prime}(\bar{x})(\cdot)(x-\bar{x}) \notin-\operatorname{int}\left(\mathscr{C}_{+}\left(T^{*}\right)\right)
$$

or

$$
\varphi_{+}^{\prime}(\bar{x})(\cdot)(x-\bar{x}) \notin-\operatorname{int}\left(\mathscr{C}_{+}\left(T^{*}\right)\right) .
$$

With [2, Lemma 4.1] or [7, Lemma 4.5], this implies that, for every $x \in S$, there exists some $\bar{\ell} \in T^{*}$ with

$$
\max \left\{-\varphi_{-}^{\prime}(\bar{x})(\bar{\ell})(x-\bar{x}), \varphi_{+}^{\prime}(\bar{x})(\bar{\ell})(x-\bar{x})\right\} \geq 0,
$$


and the first part of the assertion is shown.

(c) Since $f^{\prime}(\bar{x})$ is convex-like and the core of the order cone equals its topological interior, a topological version of Lemma 7.7 in [15] can be applied. Then there are finite signed Radon measures $\mu_{1}, \mu_{2} \in \mathscr{C}_{+}^{*}\left(T^{*}\right)$ with $\left(\mu_{1}, \mu_{2}\right) \neq\left(0_{\mathscr{C}^{*}\left(T^{*}\right)}, 0_{\mathscr{C}^{*}\left(T^{*}\right)}\right)$ and

$$
-\int_{T^{*}} \varphi_{-}^{\prime}(\bar{x})(\cdot)(x-\bar{x}) d \mu_{1}+\int_{T^{*}} \varphi_{+}^{\prime}(\bar{x})(\cdot)(x-\bar{x}) d \mu_{2} \geq 0 \text { for all } x \in S .
$$

This completes the proof.

\section{CONCLUSION}

Normed spaces seem to be the right framework for the proof of the continuity of maximal value functionals. And this is the decisive key for the proof of the Karush-Kuhn-Tucker conditions in set optimization where Lagrange multipliers are appropriate finite signed Radon measures. The duality theory in set optimization can also benefit from the knowledge of this dual space.

\section{Acknowledgments}

The author thanks Tobias Schorn for helpful discussions on the issue of this paper. Thanks also go to two anonymous referees for constructive remarks.

\section{REFERENCES}

[1] J.F. Bonnans, A. Shapiro, Perturbation Analysis of Optimization Problems, Springer, New York, 2000.

[2] J. Jahn, Karush-Kuhn-Tucker conditions in set optimization, J. Optim. Theory Appl. 172 (2017), 707-725.

[3] J.M. Borwein, J.D. Vanderwerff, Convex Functions: Constructions, Characterizations and Counterexamples, Cambridge University Press, New York, 2010.

[4] C. Zălinescu, On the differentiability of the support function, J. Global Optim. 57 (2013), 719-731.

[5] C. Zălinescu, Relations between the convexity of a set and the differentiability of its support function, Optimization 65 (2016), 651-670.

[6] I. Sadeqi, S. Hassankhali, Smulyan Lemma and Differentiability of the Support Function, Anal. Theory Appl. 34 (2018), 348-357.

[7] T. Schorn, Optimalitätsbedingungen in der Mengenoptimierung, Master Thesis, University of ErlangenNürnberg, Germany, 2017.

[8] J. Jahn, Vectorization in set optimization, J. Optim. Theory Appl. 167 (2015), 783-795.

[9] J. Diestel, Sequences and Series in Banach Spaces, Springer, New York, 1984.

[10] G. Wang, Funktionalanalysis, manuscript, University of Freiburg, Germany, 2013.

[11] F.H. Clarke, Optimization and Nonsmooth Analysis, SIAM, Philadelphia, 1990.

[12] J. Jahn, Introduction to the Theory of Nonlinear Optimization, Springer, Cham, 2020.

[13] J. Jahn, T.X.D. Ha, New order relations in set optimization, J. Optim. Theory Appl. 148 (2011), $209-236$.

[14] R.C. Young, The algebra of many-valued quantities, Math. Ann. 104 (1931), 260-290.

[15] J. Jahn, Vector Optimization - Theory, Applications, and Extensions, Springer, Berlin, 2011. 